\title{
Open access liquid chromatography/tandem mass spectrometry: implementation of a fully quantitative system in a drug discovery laboratory
}

This article was published in the following Dove Press journal:

International Journal of High Throughput Screening

19 February 2010

Number of times this article has been viewed

John G Swales

Gary Wilkinson

Richard Gallagher

Clare Hammond

Charles O'Donnell

Raimund Peter

AstraZeneca, Discovery Drug Metabolism and Pharmacokinetics, CVGI Research Area, Alderley Park, Macclesfield, Cheshire, UK
Correspondence: JG Swales AstraZeneca, Discovery Drug Metabolism and Pharmacokinetics, CVGI Research Area, Alderley Park, Macclesfield, Cheshire, SK 10 4TG, UK Email john.swales@astrazeneca.com

\begin{abstract}
High performance liquid chromatography with electrospray ionization tandem mass spectrometry (LC/ESI-MS/MS) has been utilized to produce fully open access (OA) quantitative systems. The systems described have the ability to optimize the mass spectrometer analysis method, stack these optimizations, create the mass spectrometry analytical methods and automatically process data for reporting. An in-house, Microsoft ${ }^{\circledR}$ Excel-based sample list generator in conjunction with two manufacturers' OA software packages have been implemented and are used exclusively for all in vivo and in vitro drug metabolism and pharmacokinetic assays within the department. We demonstrate that the OA systems have had a positive effect on the average turnaround times of all assays by utilizing the mass spectrometer time more effectively and by minimizing instrument downtime. Turnaround times have been monitored for 17 months post implementation of the OA systems as part of a Lean Six Sigma efficiency optimization project, and data are presented that support a sustained improvement throughout this period.
\end{abstract}

Keywords: open access, DMPK, quantitation, bioanalysis, discovery

\section{Introduction}

The aim of drug discovery is to nominate safe and effective candidate drugs (CDs) for progression through the drug development process. A study of drug attrition rates in later stages of clinical development ${ }^{1}$ revealed that a significant number of CDs fail owing to absorption, distribution, metabolism, elimination (ADME) and toxicological issues. Lead optimization initiatives ${ }^{2}$ in drug discovery are the processes that attempt to minimize these issues prior to the handover of CDs and reduce costly failures in development. Discovery drug metabolism and pharmacokinetics (DxDMPK) departments are now standard in the pharmaceutical industry and have gone someway to address the ADME attrition of CDs. DMPK departments utilize a wide variety of assays (eg, metabolic stability, permeability, time dependent inhibition) to explore the ADME profile of a particular chemical series of interest. Liquid chromatography coupled with mass spectrometry on triple quadrupole instruments operating in selected reaction monitoring (SRM) mode has become the analytical method of choice for DMPK analysis. ${ }^{3}$ This method offers high sensitivity, specificity, selectivity and rapid turnaround in a high throughput environment making it ideal for quantitation of low levels of analyte in the presence of biological endogenous material.

The DxDMPK section within the Cardiovascular and Gastrointestinal research area at AstraZeneca, Alderley Park has utilized Lean Six Sigma analysis ${ }^{4}$ (LSS) to 
improve assay turnaround times by removing non-value activities. LSS is data driven and as such a period of data collection known as the 'measure phase' is employed to build an understanding of the current process under review. Subsequent data are analyzed to find the root cause of any 'blockers' to efficiency which were highlighted in the measure phase. During our initial LSS project aimed at improving the process of gathering in vivo pharmacokinetic (PK) data it was evident that mass spectrometer use and robustness was a major 'blocker' to improving the efficiency of the process.

Open access (OA) mass spectrometry combined with generic chromatography systems and standardized sample preparation was identified as a way of managing sample throughput in a more efficient manner. OA has been used in high throughput chemistry laboratories for many years, ${ }^{5,6}$ essentially employing walk-up single quadrupole or time of flight instruments that confirm mass and give some indication of purity and structural confirmation. OA systems in quantitative environments have been described in the literature ${ }^{7}$ but fall short of 'true' OA in so much as they lack the ability for multiple users to optimize mass spectrometer methods, to stack optimization methods, create SRM methods and automatically process data for reporting. Two manufacturers now offer integrated OA software solutions that can be utilized in a quantitative way (Figure 1). Thermo Fisher Scientific (Hemel Hempstead, UK) market software called QuickQuan ${ }^{\mathrm{TM}}$ and QuickCalc $^{\mathrm{TM}}$ that enables OA sample analysis on their series of triple quadrupole mass spectrometers. Waters Corporation (Elstree, UK) market software known as OpenLynx ${ }^{\mathrm{TM}}$ and QuanBrowser ${ }^{\mathrm{TM}}$ that enables OA sample analysis on their range of mass spectrometers. Both manufacturers OA platforms are in use in our laboratories and are used exclusively for all in vivo and in vitro analysis within the department.

\section{Experimental}

\section{Liquid chromatography-mass spectrometry instrumentation}

Four different liquid chromatography/tandem mass spectrometry (LC-MS/MS) systems are used within our laboratories.

Mass spectrometers I and II: TSQ Quantum Vantage and TSQ Quantum Ultra (Thermo Fisher Scientific, Hemel Hempstead, UK) triple quadrupole mass spectrometers using an electrospray ionization source (HESI2 ${ }^{\mathrm{TM}}$, High Efficiency ESI).

Mass spectrometers III and IV: Quattro Ultima and Quattro Ultima Pt (Waters Corporation, Elstree, UK), using an electrospray ionization source.

Mass spectrometers I and II were both fitted with a Surveyor MS Pump Plus HPLC pump, (Thermo Fisher Scientific, Hemel Hempstead, UK) and a CTC Analytics HTS PAL autosampler (Presearch Ltd, Basingstoke, UK). Each autosampler was fitted with three Vici Cheminert valves (Vici AG International, Schenkon, Switzerland). Valve I is used for analytical batch injection, valve II is used for optimization infusion and valve III is used for analytical column selection. A further switching valve located on the mass spectrometer is used to divert the LC flow to waste for the initial one minute of each injection in order to protect the MS source from contamination. All valve positions and instrument parameters were controlled by ThermoFisher Xcalibur $^{\mathrm{TM}}$ software, version 2.0.0 (Thermo Fisher Scientific, Hemel Hempstead, UK).

Mass spectrometers III and IV were both fitted with a degasser unit, HPLC pump and column oven Agilent 1100 series (Agilent Ltd, Cheadle, UK), comprising a degasser (model G1322A), binary pump (model G1312A) and column switching module (G1316A). The column switching module is used for analytical column selection. Samples are

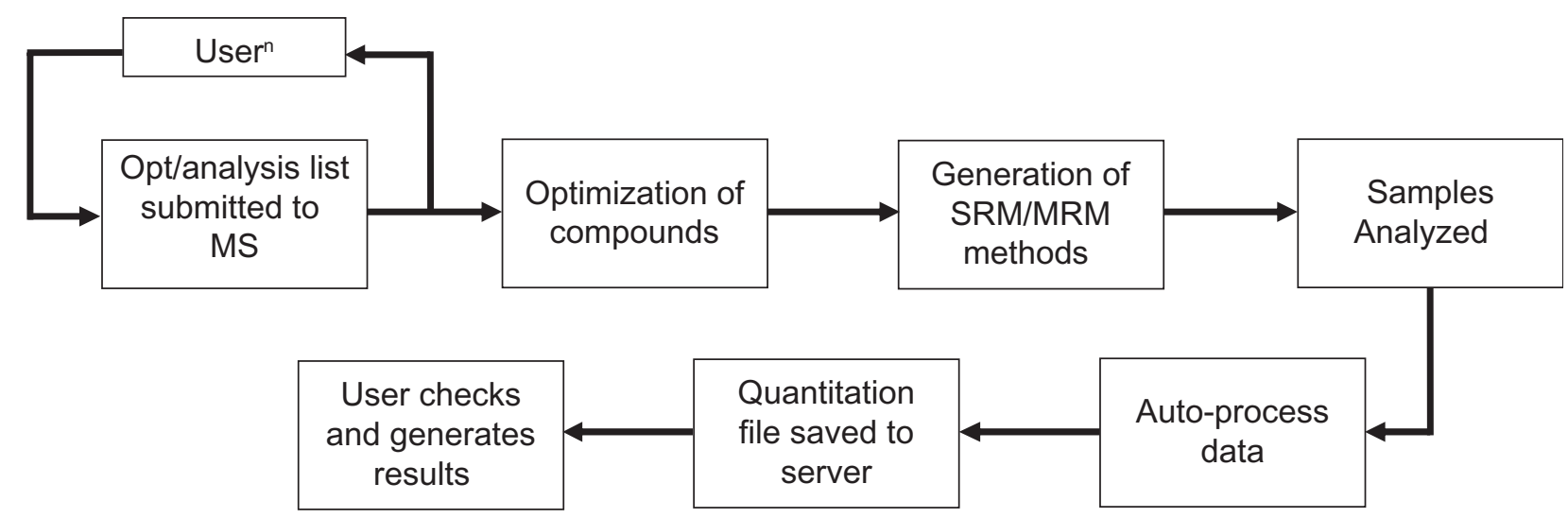

Figure I A schematic of the open access work flow. Each box shows a fully automated step in the open access process. 
introduced into the system via a CTC Analytics HTS PAL autosampler (Presearch Ltd, Basingstoke, UK). A further switching valve located on the mass spectrometer is used to divert the LC flow to waste for the initial one minute of each injection in order to protect the MS source from contamination. All valve positions and instrument parameters were controlled by Waters MassLynx ${ }^{\mathrm{TM}}$ software, version 4.1 (Waters Corporation, Elstree, UK).

\section{Open access software}

Open Access sample login and data processing on the Thermo Fisher Scientific mass spectrometer systems was performed using the software packages Thermo Fisher Scientific QuickQuan $^{\mathrm{TM}}$ (version 2.1) and QuickCalc ${ }^{\mathrm{TM}}$ (version 6.0.3). Open Access sample login and data processing on the Waters Corporation mass spectrometer systems was performed using the Waters Corporation software package OpenLynx ${ }^{\mathrm{TM}}$ (version 4.1), QuanLynx ${ }^{\mathrm{TM}}$ (version 4.0)

\section{Open access liquid chromatography-mass spectrometry analysis methods}

Four experimental methods (I to IV) are available by utilizing two linear gradient methods on two different columns: (1) Synergi Max RP (Phenomenex, Macclesfield, UK), $50 \mathrm{~mm} \times$ $2.1 \mathrm{~mm}, 5 \mu \mathrm{m}$ particle size, (2) Hypersil Gold C18 (Thermo Fisher Scientific, Loughborough, UK), $30 \mathrm{~mm} \times 2.1 \mathrm{~mm}$, $5 \mu \mathrm{m}$ particle size. The methods offer the versatility to analyze a wide variety of drug candidates and maintain the balance between speed and chromatographic separation that is required in a high throughput environment. ${ }^{8,9}$

Gradient system I uses column (1) with mobile phases (A) $10 \mathrm{mM}$ ammonium acetate in water and (B) $10 \mathrm{mM}$ ammonium acetate in methanol. The linear gradient used is ( $\mathrm{T}=$ minutes): at $\mathrm{T}=0.0,95 \% \mathrm{~A}: 5 \% \mathrm{~B}, \mathrm{~T}=3.0,5 \% \mathrm{~A}: 95 \%$ $\mathrm{B}, \mathrm{T}=4.0,5 \% \mathrm{~A}: 95 \% \mathrm{~B}, \mathrm{~T}=4.1,95 \% \mathrm{~A}: 5 \% \mathrm{~B}, \mathrm{~T}=5.0,95 \%$ $\mathrm{A}: 5 \% \mathrm{~B}$. This system is the preferred analytical method for all in vivo analysis within the department.

Gradient system II uses column (1) with mobile phases (C) $0.1 \%(\mathrm{v} / \mathrm{v})$ formic acid in water and (D) $0.1 \%(\mathrm{v} / \mathrm{v})$ formic acid in methanol. The linear gradient used is ( $\mathrm{T}=$ minutes): at $\mathrm{T}=0.0,95 \% \mathrm{C}: 5 \% \mathrm{D}, \mathrm{T}=3.0,5 \% \mathrm{C}: 95 \% \mathrm{D}, \mathrm{T}=4.0,5 \%$ $\mathrm{C}: 95 \% \mathrm{D}, \mathrm{T}=4.1,95 \% \mathrm{C}: 5 \% \mathrm{D}, \mathrm{T}=5.0,95 \% \mathrm{C}: 5 \% \mathrm{D}$. This system is used for compounds that fail to give an adequate mass spectrometric response with gradient system I.

Gradient method III uses column (2) with mobile phase (C) and (D). The linear gradient used is ( $\mathrm{T}=$ minutes): $\mathrm{T}=0.0$, 96\% C:4\% D, T = 1.0, 10\% C:90\% D, T $=1.9,10 \% \mathrm{C}: 90 \%$ $\mathrm{D}, \mathrm{T}=2.0,96 \% \mathrm{C}: 4 \% \mathrm{D}, \mathrm{T}=2.5,96 \% \mathrm{C}: 4 \% \mathrm{D}$. This system is the preferred analytical method for all in vitro analysis within the department.

Gradient method IV uses column (2) with mobile phase (A) and (B). The linear gradient used is ( $\mathrm{T}=$ minutes $): \mathrm{T}=$ $0.0,96 \% \mathrm{~A}: 4 \% \mathrm{~B}, \mathrm{~T}=1.0,10 \% \mathrm{~A}: 90 \% \mathrm{~B}, \mathrm{~T}=1.9,10 \%$ $\mathrm{A}: 90 \% \mathrm{~B}, \mathrm{~T}=2.0,96 \% \mathrm{~A}: 4 \% \mathrm{~B}, \mathrm{~T}=2.5,96 \% \mathrm{~A}: 4 \% \mathrm{~B}$. This system is rarely used but offers rapid analysis at $\mathrm{pH} 7$ should this be required.

All of the systems use a flow rate of $0.75 \mathrm{~mL} / \mathrm{min}$ and an injection volume of $50 \mu \mathrm{L}$ are used.

\section{Generic sample preparation}

A generic sample preparation method has been introduced within the department with the intention of reducing variability, reducing instrument downtime and improving the quality of the data generated on the instruments.

$50 \mu \mathrm{L}$ of sample matrix from in vivo studies is protein precipitated with $200 \mu \mathrm{L}$ of cold acetonitrile $\left(4^{\circ} \mathrm{C}\right)$ containing internal standard. The internal standard is project specific and chosen to represent the chemical series under investigation. The samples are then centrifuged at $3700 \mathrm{~g}$ for 10 minutes. $50 \mu \mathrm{L}$ of the resulting supernatant is then diluted with $300 \mu \mathrm{L}$ of deionized water prior to injection onto the OA systems.

Experiments involving in vitro incubations are halted by addition of cold acetonitrile $\left(4^{\circ} \mathrm{C}\right)$ containing internal standard. The resulting mixture is then centrifuged $(3700 \mathrm{~g})$ to remove any protein. $100 \mu \mathrm{L}$ of supernatant is diluted with $100 \mu \mathrm{L}$ of deionized water prior to injection onto the OA systems. All in vitro incubations were performed on a Tecan Genesis 200 (Tecan UK Limited, Reading, UK).

\section{Quality control test solution preparation}

A $1 \mathrm{mg}$ weighing of erythromycin, lidocaine, propanolol and warfarin, (Sigma Aldrich, Poole, Dorset, UK) were individually dissolved in $1 \mathrm{~mL}$ of deionized water to form stock solutions. $50 \mu \mathrm{L}$ of erythromycin, propanolol and warfarin stock solutions and $5 \mu \mathrm{L}$ of lidocaine stock solution were then mixed and diluted with $1 \mathrm{~L}$ of deionized water to give a final concentration of $50 \mathrm{ng} / \mathrm{mL}$ erythromycin, propanolol, warfarin and $5 \mathrm{ng} / \mathrm{mL}$ lidocaine. $50 \mu \mathrm{L}$ of this solution was injected onto the systems as a quality control between analytical batches.

\section{Results and discussion OA using QuickQuan ${ }^{T M}$ and QuickCalc ${ }^{T M}$} The two Thermo Fisher Scientific TSQ Quantum mass spectrometers each function as identical OA systems. We have constructed a Microsoft ${ }^{\circledR}$ Excel 2003 (Microsoft 
Corp, Seattle, USA) based workbook that is accessed via users office based workstations to enable easy construction of both optimization and analysis run lists. The workbook consists of two worksheets (Figure 2a and 2b). Optimization worksheet is populated with compound identification numbers. If compounds are in a cassette format they can be grouped into named drug sets which will be used to group SRM transitions post optimization. The optimization solution locations are also recorded on this worksheet (96-well plate format). The generic chromatography methods, injection volume and use of an internal standard are selected from drop down menus, the default settings are gradient system I with a $50 \mu \mathrm{L}$ injection volume and inclusion of an internal standard as this is the most popular configuration and minimizes user input. The analysis worksheet is populated by the user with the sample injection order and other critical information such as drug set name, user specified
(A)

\begin{tabular}{|c|c|c|c|c|c|c|c|}
\hline & $\bar{A}$ & $\bar{B}$ & $\overline{\mathrm{C}}$ & $\overline{\mathrm{D}}$ & $E$ & G & $\mathrm{H}$ \\
\hline 1 & Compound & Formula & Drugset Name & Well No & & & \\
\hline 2 & Compound 1 & $\mathrm{C} 16 \mathrm{H} 13 \mathrm{~N} 2$ & TestSetA & A1 & & Fill in the relevant information & \\
\hline 3 & Compound 2 & C15H10N2 & TestSetA & A2 & & & \\
\hline 4 & Compound 3 & $\mathrm{C} 22 \mathrm{H} 29 \mathrm{~N} 2 \mathrm{O}$ & TestSetA & A3 & & & \\
\hline 5 & Compound 4 & $\mathrm{C} 21 \mathrm{H} 27 \mathrm{~N} 2$ & TestSetA & A4 & & Get Formulas & \\
\hline 6 & Compound 5 & $\mathrm{C} 19 \mathrm{H} 23 \mathrm{~N} 2$ & TestSetA & A5 & & & \\
\hline 7 & Compound 6 & $\mathrm{C} 18 \mathrm{H} 15 \mathrm{~N} 2 \mathrm{O}$ & TestSetB & A6 & & & \\
\hline 8 & Compound 7 & $\mathrm{C} 16 \mathrm{H} 12 \mathrm{NS}$ & TestSetB & A7 & & & \\
\hline 9 & Compound 8 & $\mathrm{C} 18 \mathrm{H} 15 \mathrm{~N} 2 \mathrm{O} 2$ & TestSetB & A8 & & & \\
\hline 10 & Compound 9 & $\mathrm{C} 17 \mathrm{H} 13 \mathrm{~N} 2 \mathrm{O} 2$ & TestSetB & A9 & & Export Runlists & \\
\hline 11 & Compound 10 & $\mathrm{C} 19 \mathrm{H} 17 \mathrm{~N} 2 \mathrm{O} 2$ & TestSetB & A10 & & & \\
\hline 12 & Compound 11 & $\mathrm{C} 21 \mathrm{H} 23 \mathrm{~N} 2 \mathrm{O} 3$ & TestSetC & A11 & & & \\
\hline 13 & Compound 12 & C17H15N2 & TestSetC & A12 & & & \\
\hline 14 & Compound 13 & $\mathrm{C} 17 \mathrm{H} 15 \mathrm{~N} 2 \mathrm{O}$ & TestSetC & A13 & & Select LC Method for run: & \\
\hline 15 & Compound 14 & $\mathrm{C} 19 \mathrm{H} 18 \mathrm{~N} 3 \mathrm{O} 2$ & TestSetC & A14 & & $5 \mathrm{~min}$ Acetate & \\
\hline 16 & Compound 15 & C17H15N2 & TestSetC & A15 & & & \\
\hline 17 & Compound 16 & C17H14BrN2 & TestSetD & A16 & & Select: Use internal standard: & \\
\hline 18 & Compound 17 & $\mathrm{C} 23 \mathrm{H} 17 \mathrm{~N} 2 \mathrm{O}$ & TestSetD & A17 & & Yes & \\
\hline 19 & Compound 18 & C23H19N2 & TestSetD & A18 & & & \\
\hline 20 & Compound 19 & $\mathrm{C} 16 \mathrm{H} 12 \mathrm{BrN} 2$ & TestSetD & A19 & & Select inject volume & \\
\hline 21 & Compound 20 & C16H14N3 & TestSetD & A20 & & $50 \mathrm{\mu l}$ & \\
\hline 22 & Compound 21 & $\mathrm{C} 2 \mathrm{OH} 2 \mathrm{ON} 3 \mathrm{O}$ & TestSetE & A21 & & & \\
\hline 23 & Compound 22 & $\mathrm{C} 31 \mathrm{H} 20 \mathrm{~N} 6 \mathrm{O}$ & TestSetE & A22 & & Study name: & \\
\hline 24 & Compound 23 & $\mathrm{C} 2 \mathrm{OH} 18 \mathrm{~N} 3 \mathrm{O} 2$ & TestSetE & A23 & & Test Study & \\
\hline 25 & Compound 24 & $\mathrm{C} 18 \mathrm{H} 17 \mathrm{~N} 2$ & TestSetE & A24 & & & \\
\hline 26 & Compound 25 & C19H19N2 & TestSetE & A25 & & & \\
\hline 27 & Compound 26 & $\mathrm{C} 18 \mathrm{H} 16 \mathrm{~N} 2$ & TestSetF & A26 & & & \\
\hline 28 & Compound 27 & $\mathrm{C} 18 \mathrm{H} 14 \mathrm{BrN} 2 \mathrm{O}$ & TestSetF & A27 & & & \\
\hline 29 & Compound 28 & $\mathrm{C} 19 \mathrm{H} 15 \mathrm{~N} 2 \mathrm{O} 3$ & TestSetF & A28 & & & \\
\hline 30 & Compound 29 & $\mathrm{C} 22 \mathrm{H} 27 \mathrm{~N} 4$ & TestSetF & A29 & & & \\
\hline 31 & Compound 30 & $\mathrm{C} 2 \mathrm{OH} 17 \mathrm{BrN} 3 \mathrm{O} 2$ & TestSetF & A30 & & & \\
\hline 32 & Compound 31 & $\mathrm{C} 17 \mathrm{H} 12 \mathrm{BrN} 2 \mathrm{O} 2$ & TestSetG & A31 & & & \\
\hline 33 & Compound 32 & $\mathrm{C} 19 \mathrm{H} 14 \mathrm{BrN} 2 \mathrm{O} 3$ & TestSetG & A32 & & & \\
\hline 34 & Compound 33 & $\mathrm{C} 23 \mathrm{H} 16 \mathrm{BrN} 2 \mathrm{O}$ & TestSetG & A33 & & & \\
\hline 35 & Compound 34 & $\mathrm{C} 15 \mathrm{H} 10 \mathrm{~N} 2 \mathrm{O}$ & TestSetG & A34 & & & \\
\hline 36 & Compound 35 & $\mathrm{C} 32 \mathrm{H} 45 \mathrm{~N} 2$ & TestSetG & A35 & & & \\
\hline 37 & Compound 36 & $\mathrm{C} 29 \mathrm{H} 37 \mathrm{~N} 2 \mathrm{O} 2$ & TestSetH & A36 & & & \\
\hline 38 & Compound 37 & $\mathrm{C} 24 \mathrm{H} 29 \mathrm{~N} 3 \mathrm{O} 2$ & TestSetH & A37 & & & \\
\hline 39 & Compound 38 & $\mathrm{C} 10 \mathrm{H} 9 \mathrm{NO}$ & TestSetH & A38 & & & \\
\hline 40 & Compound 39 & $\mathrm{C} 10 \mathrm{H} 9 \mathrm{NO}$ & TestSetH & A39 & & & \\
\hline 41 & Compound 40 & C11H11NO & TestSetH & A40 & & & \\
\hline 42 & Compound 41 & C9H7NO & TestSetl & A41 & & & \\
\hline 43 & Compound 42 & C11H19NO2 & TestSetl & A42 & & & \\
\hline 44 & Compound 43 & $\mathrm{C} 12 \mathrm{H} 12 \mathrm{O} 6$ & TestSetl & A43 & & & \\
\hline & Compound 44 & C16H12N2 & TestSetl & A44 & & & \\
\hline
\end{tabular}




\begin{tabular}{|c|c|c|c|c|c|c|}
\hline 4 & A & $B$ & C & D & $E$ & T \\
\hline 1 & Bracket Type $=2$ & & & & & \\
\hline 2 & Sample Type & Sample Name & Level & Position & 'L5 & \\
\hline 3 & Blank & wash & 1 & $1: 1$ & TestSetA & \\
\hline 4 & Blank & wash & & $1: 2$ & TestSetA & \\
\hline 5 & Blank & wash & & $1: 3$ & TestSetA & \\
\hline 6 & Blank & wash & & $1: 4$ & TestSetA & \\
\hline 7 & Blank & wash & & $1: 5$ & TestSetA & \\
\hline 8 & Blank & Zero & & $1: 6$ & TestSetA & \\
\hline 9 & Std Update & Calibration 1 & 1 & $1: 7$ & TestSetA & \\
\hline 10 & Std Update & Calibration 5 & 5 & $1: 8$ & TestSetA & \\
\hline 11 & Std Update & Calibration 10 & 10 & $1: 9$ & TestSetA & \\
\hline 12 & Std Update & Calibration 50 & 50 & $1: 10$ & TestSetA & \\
\hline 13 & Std Update & Calibration 100 & 100 & 1:11 & TestSetA & \\
\hline 14 & Std Update & Calibration 500 & 500 & 1:12 & TestSetA & \\
\hline 15 & Std Update & Calibration 1000 & 1000 & $1: 13$ & TestSetA & \\
\hline 16 & Std Update & Calibration 2000 & 2000 & $1: 14$ & TestSetA & \\
\hline 17 & Std Update & Calibration 5000 & 5000 & 1:15 & TestSetA & \\
\hline 18 & Std Update & Calibration 10000 & 10000 & 1:16 & TestSetA & \\
\hline 19 & Blank & Blank & & 1:17 & TestSetA & \\
\hline 20 & Blank & Blank & & 1:18 & TestSetA & \\
\hline 21 & Unknown & Compound $1 \mathrm{~N} 11$ & & $1: 19$ & TestSetA & \\
\hline 22 & Unknown & Compound $1 \mathrm{~N} 12$ & & $1: 20$ & TestSetA & \\
\hline 23 & Unknown & Compound $1 \mathrm{~N} 13$ & & $1: 21$ & TestSetA & \\
\hline 24 & Unknown & Compound $1 \mathrm{~N} 14$ & & $1: 22$ & TestSetA & \\
\hline 25 & Unknown & Compound $1 \mathrm{~N} 15$ & & $1: 23$ & TestSetA & \\
\hline 26 & Unknown & Compound $1 \mathrm{~N} 16$ & & $1: 24$ & TestSetA & \\
\hline 27 & Unknown & Compound $1 \mathrm{~N} 17$ & & $1: 25$ & TestSetA & \\
\hline 28 & Unknown & Compound $1 \mathrm{~N} 18$ & & $1: 26$ & TestSetA & \\
\hline 29 & Blank & Blank & & $1: 27$ & TestSetA & \\
\hline 30 & Unknown & Compound $1 \mathrm{~N} 21$ & & $1: 28$ & TestSetA & \\
\hline 31 & Unknown & Compound $1 \mathrm{~N} 22$ & & $1: 29$ & TestSetA & \\
\hline 32 & Unknown & Compound $1 \mathrm{~N} 23$ & & $1: 30$ & TestSetA & \\
\hline 33 & Unknown & Compound $1 \mathrm{~N} 24$ & & 1:31 & TestSetA & \\
\hline 34 & Unknown & Compound $1 \mathrm{~N} 25$ & & $1: 32$ & TestSetA & \\
\hline 35 & Unknown & Compound $1 \mathrm{~N} 26$ & & $1: 33$ & TestSetA & \\
\hline 36 & Unknown & Compound $1 \mathrm{~N} 27$ & & $1: 34$ & TestSetA & \\
\hline 37 & Unknown & Compound $1 \mathrm{~N} 28$ & & $1: 35$ & TestSetA & \\
\hline 38 & Blank & Blank & & $1: 36$ & TestSetA & \\
\hline 39 & Unknown & Compound 1 PO1 1 & & $1: 37$ & TestSetA & \\
\hline 40 & Unknown & Compound 1 PO1 2 & & $1: 38$ & TestSetA & \\
\hline 41 & Unknown & Compound 1 PO1 3 & & $1: 39$ & TestSetA & \\
\hline 42 & Unknown & Compound 1 PO1 4 & & $1: 40$ & TestSetA & \\
\hline 43 & Unknown & Compound 1 PO1 5 & & $1: 41$ & TestSetA & \\
\hline 44 & Unknown & Compound 1 PO1 6 & & 1:42 & TestSetA & \\
\hline 45 & Unknown & Compound 1 PO1 7 & & $1: 43$ & TestSetA & \\
\hline 46 & Unknown & Compound 1 PO1 8 & & $1: 44$ & TestSetA & \\
\hline 47 & Blank & Blank & & $1: 45$ & TestSetA & \\
\hline 48 & Unknown & Compound $1 \mathrm{PO} 21$ & & $1: 46$ & TestSetA & \\
\hline 49 & Unknown & Compound 1 PO2 2 & & $1: 47$ & TestSetA & \\
\hline 50 & Unknown & Compound 1 PO2 3 & & $1: 48$ & TestSetA & \\
\hline 51 & Unknown & Compound 1 PO2 4 & & $1: 49$ & TestSetA & \\
\hline 52 & Unknown & Compound 1 PO2 5 & & $1: 50$ & TestSetA & \\
\hline 53 & Unknown & Compound 1 PO2 6 & & $1: 51$ & TestSetA & \\
\hline 54 & Unknown & Compound 1 PO2 7 & & 1:52 & TestSetA & \\
\hline 55 & Unknown & Compound 1 PO2 8 & & $1: 53$ & TestSetA & \\
\hline & $4 \cdot 1$ & ion Analysis & & & & \\
\hline
\end{tabular}

Figure 2A A representation of the Optimization worksheet in the Excel-based sample list generator. Figure 2B:A representation of the Analysis worksheet in the Excel-based sample list generator.

sample location and nominal concentration. Samples are designated as blanks, standards, quality controls or unknowns. Once the analysis worksheet is constructed, the user clicks the 'Get Formulas' button which downloads the molecular formula from the company database. The user then clicks the 'Export Runlists' button on the optimization worksheet. The software checks for inconsistencies and population errors between the two worksheets, if any errors are detected they are highlighted on the worksheets and must be corrected before they can be saved. If no errors are detected the user is prompted for a unique file identifier and the optimization and analysis worksheets are saved as text (.txt) and comma delimited (.csv) file formats respectively.
Submission to the instrument sample queue is performed by the user via QuickQuan ${ }^{\mathrm{TM}}$. In the optimization view (Figure 3a) the optimization list can be imported, populating the database with compound identifiers (name and mass), drugset and the location of the compound optimization solution. This is visualized on a virtual 96-well plate. The analysis list is imported into the acquisition view (Figure 3b), here an internal standard can be specified and an output path for storage of the acquired data files can be input. The user clicks the 'go' button which opens a final option window where parameters such as ionization method, polarity, instrument settings and any post analysis processing methods are selected. Clicking 'Run' switches on the mass spectrometer and all peripherals and initiates the optimization sequence. 


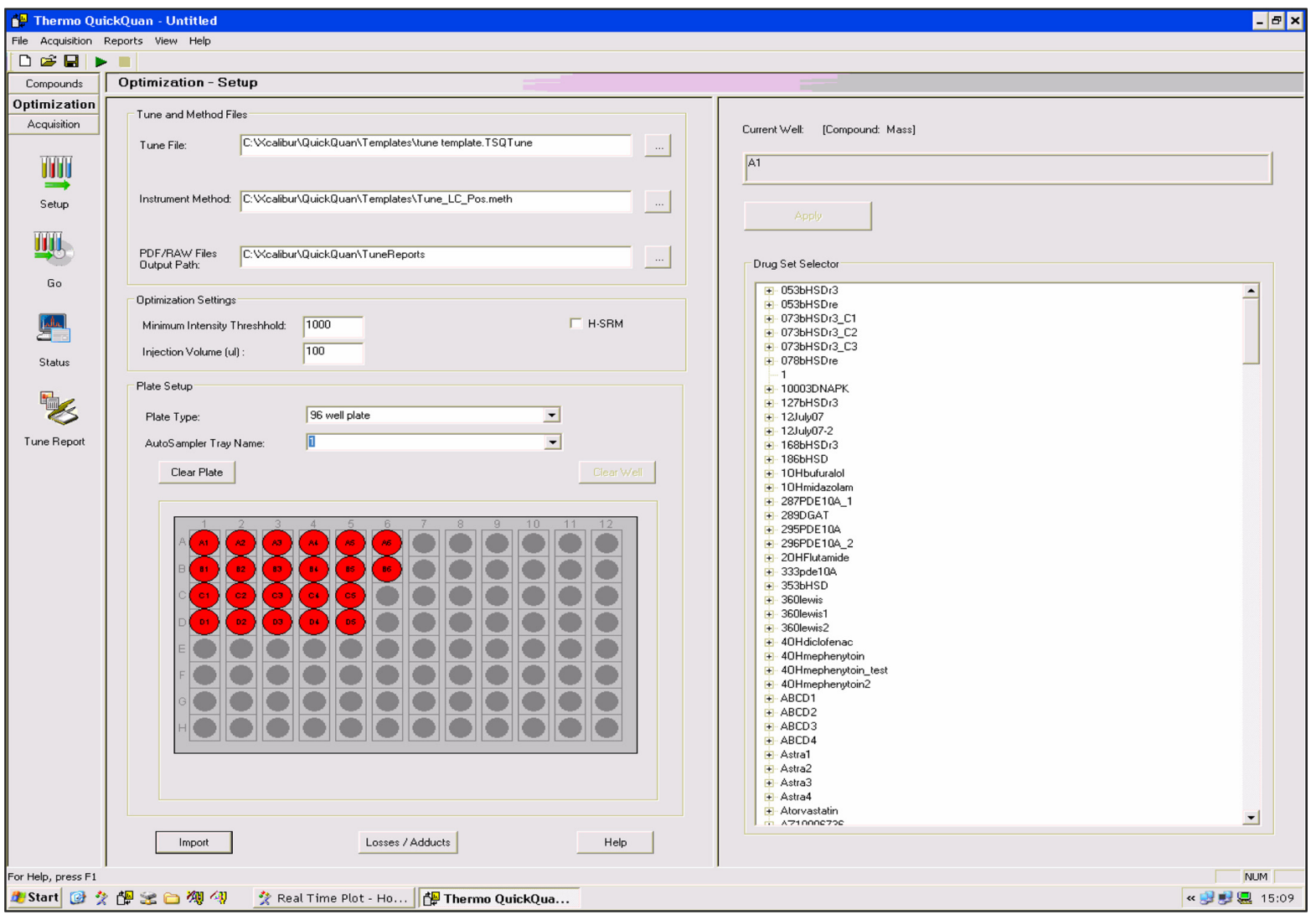

Figure 3A A screenshot of the Optimization view within the QuickQuan ${ }^{\mathrm{TM}}$ software.

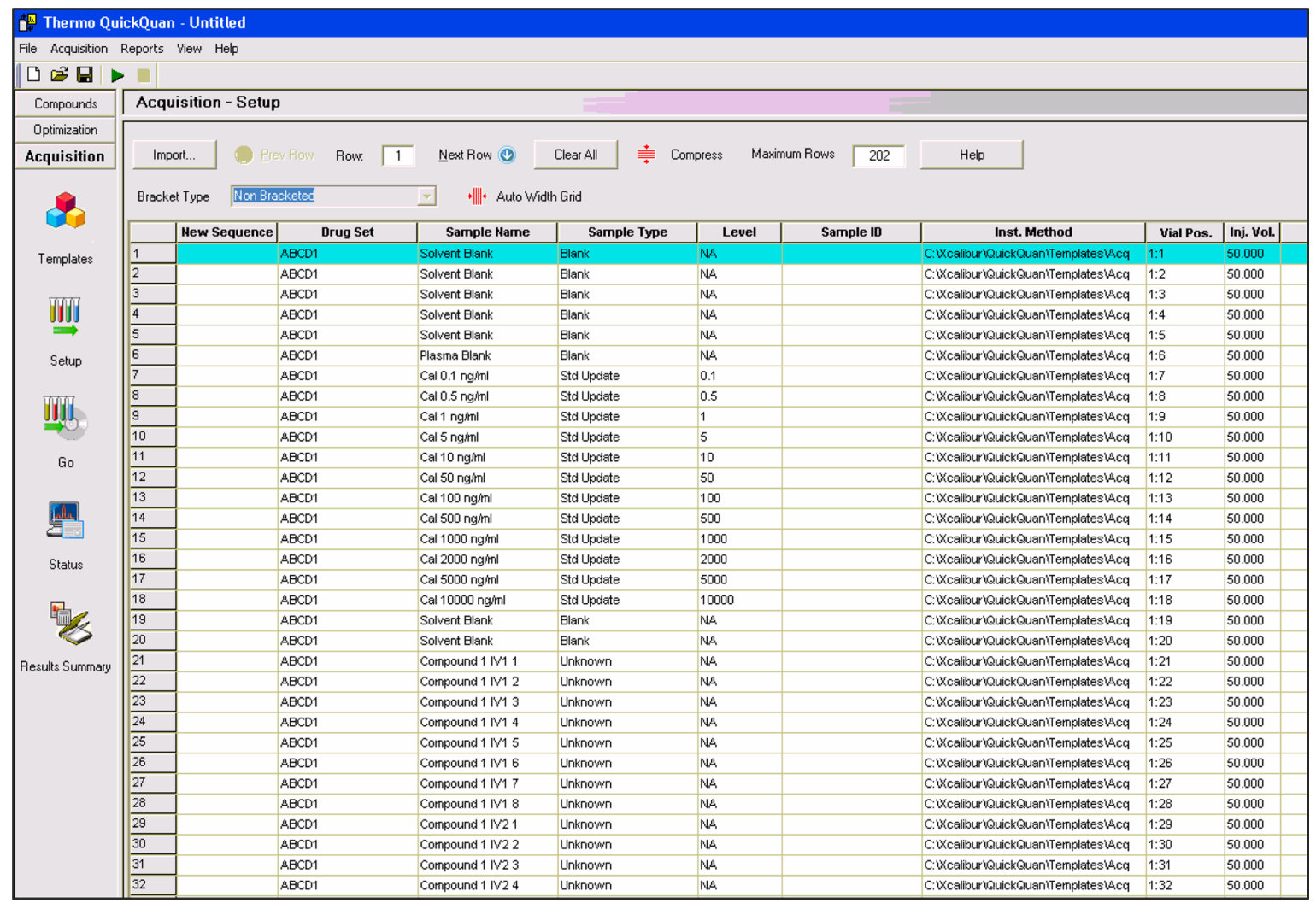

Figure 3B A screenshot of the Acquisition view within the QuickQuan ${ }^{\top M}$ software. 
QuickQuan Compound Optimization Result Report - Verapamil

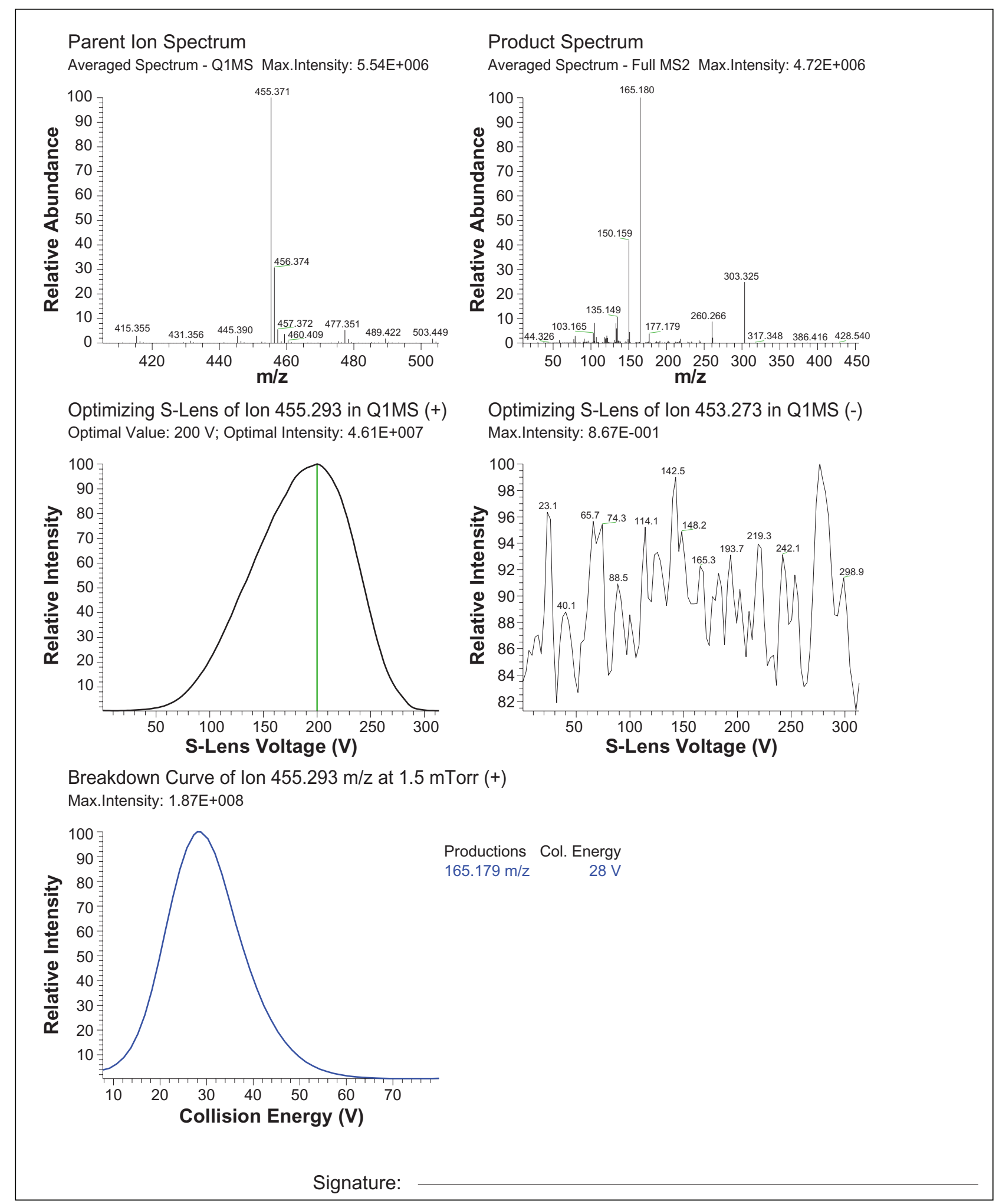

Figure 4 An example of a QuickQuan ${ }^{T M}$ Optimization Tune Report for verapamil (Continued on p. 8). 
QuickQuan Compound Optimization Result Report - Verapamil

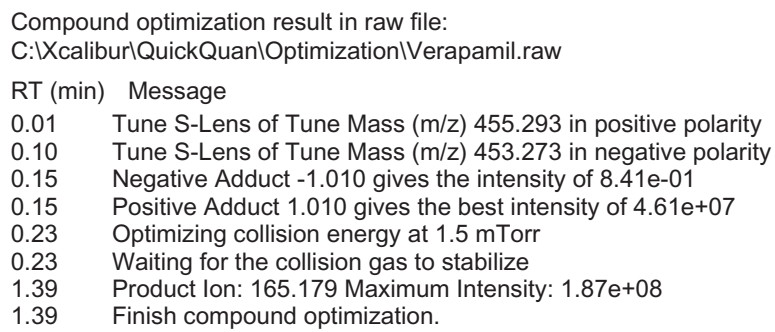

Signature:

Figure 4 (Continued).

Any subsequent users open a new instance of QuickQuan ${ }^{\mathrm{TM}}$ and repeat the process.

\section{Thermo Fisher Scientific instrument optimization procedure}

The QuickQuan ${ }^{\mathrm{TM}}$ optimization procedure infuses a standard $1 \mu \mathrm{g} / \mathrm{mL}$ methanolic solution of each compound (or a mixture of compounds) via the syringe on the autosampler into the mobile phase eluent $(250 \mu \mathrm{L} / \mathrm{min})$ directed to the mass spectrometer source. The infusion lasts for a total of 1 min during which an optimization algorithm is run. QuickQuan ${ }^{\mathrm{TM}}$ uses the molecular formula uploaded by the user to calculate a monoisotopic molecular mass (M) and scans a mass window $\mathrm{M} \pm 50$ amu. Parent ions are scanned in positive and negative ion mode against a tube lens ramp ( 0 to 300 volts). The algorithm selects polarity and tube lens voltage giving the most intense parent; it then records the measured molecular ion. Parent mass and tube lens voltage are applied to quadrupole one and a breakdown curve is acquired across a collision energy ramp (5 to 80 volts) with the collision cell at a pressure of 1.5 mTorr argon. The most intense product ion and corresponding collision voltage is recorded. Polarity, parent ion, tube lens voltage, product ion and collision energy are saved to the QuickQuan ${ }^{\mathrm{TM}}$ database for future use. A detailed report is also constructed (Figure 4) showing parent/product ion spectra, useful for investigating failed optimizations. The database storing all of the optimized parameters is located on a company server accessible from all TSQ Quantum instruments in the laboratory, removing the need to optimize compounds more than once.

QuickQuan ${ }^{\mathrm{TM}}$ uses the saved parameters to construct SRM methods for a compound post optimization for use in the analytical batch.

\section{Thermo Fisher Scientific instrument data processing}

QuickQuan $^{\mathrm{TM}}$ saves all of the data captured for a batch in a user identified output path. Each log in procedure is given a time/date stamp and an Xcalibur sequence file (.sld file) is created for the analysis. Users access the .sld file at their office workstations using QuickCalc ${ }^{\mathrm{TM}}$. QuickCalc ${ }^{\mathrm{TM}}$ is a suite of programs specifically designed for high throughput DMPK mass spectrometric analysis. ${ }^{10}$ It encompasses software solutions for hepatic clearance, permeability, drug-drug interactions and pharmacokinetics, although at this time the authors only use the generic chromatography viewer that allows quantification of analytical data. QuickCalc ${ }^{\mathrm{TM}}$ autoprocesses the analytical batch; integration is based on a user specified standard within the run and can be easily checked by the user (Figure 5a and 5b). Designated standards, quality controls, blanks, unknowns and nominal concentrations are imported into QuickQuan ${ }^{\mathrm{TM}}$ at the beginning of the $\log$ on process, these are embedded in the raw data and are used in QuickCalc ${ }^{\mathrm{TM}}$ for quantification. Standards can be included or excluded from the calibration curve and different analytes in a drug set can be selected for processing.

Results are reported through a report generator that can be custom formatted to the users requirements, result summaries are displayed in Excel. The different software suites within QuickCalc ${ }^{\mathrm{TM}}$ have the scope to report DMPK specific data as a finished product.

\section{Open access using OpenLynx $x^{T M}$ and Quan Browser ${ }^{\mathrm{TM}}$}

Our department has two Quattro Ultima mass spectrometers (one Quattro Ultima, one Quattro Ultima Pt) each functioning as identical OA systems and running the same analytical methods as the Thermo equipment. The department uses a similar custom in-house Excel based 'run list generator' to that used with the QuickQuan ${ }^{\mathrm{TM}}$ software. Files are saved to the same server formatted (as .txt files) for import into the 

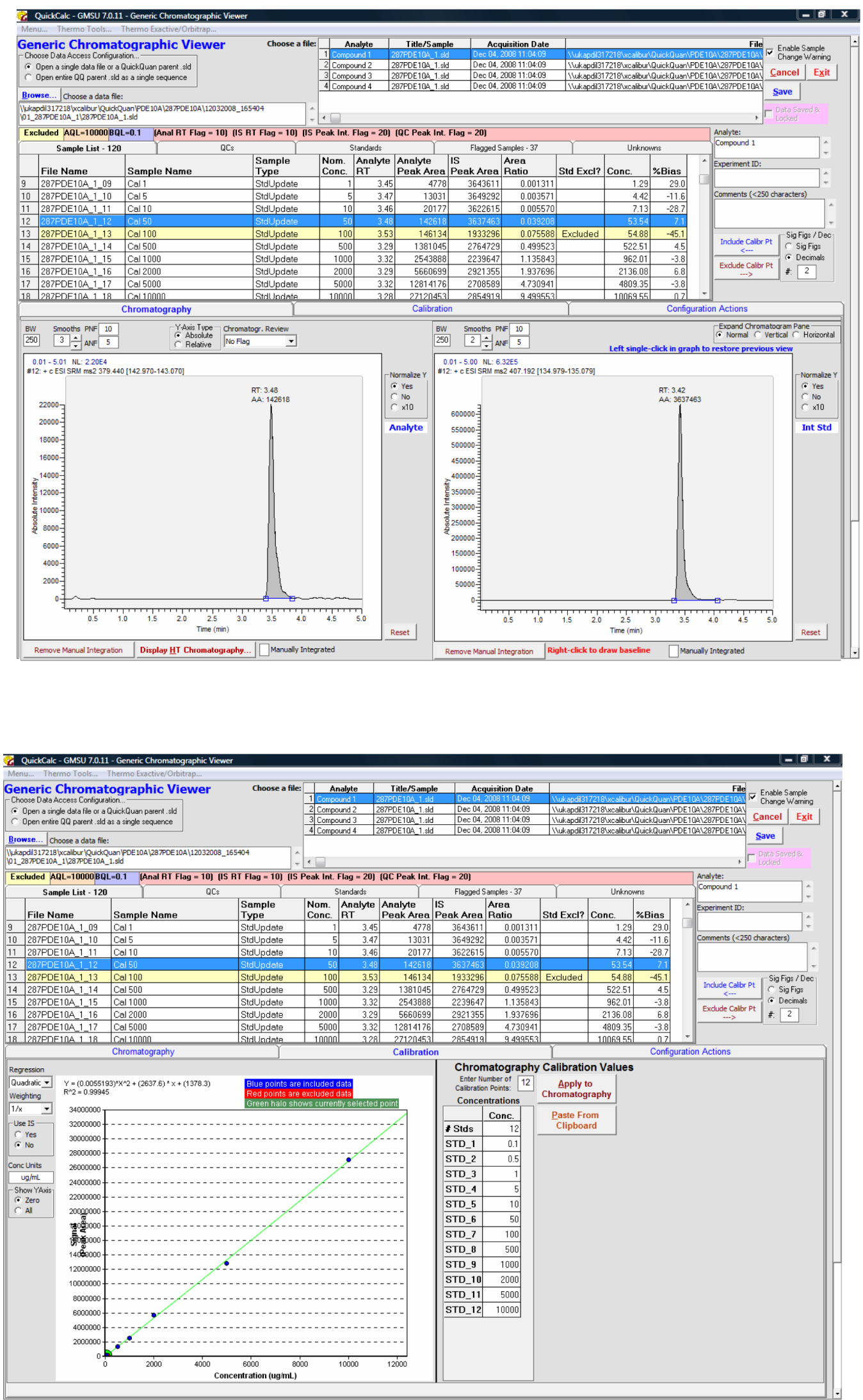

Figure 5A and 5B The QuickCalc ${ }^{T M}$ generic chromatography viewer user interface developed by Gubbs Inc. The software auto processes data acquired on the thermo Fisher Scientific instruments across the computer network. 
OpenLynx ${ }^{\mathrm{TM}}$ software. The log in procedure is administrated through an OpenLynx ${ }^{\mathrm{TM}} \log$ in console on the desktop of the instrument linked workstation. The console is left open along with a batch manager that monitors the sample queue.

The console acts as a wizard prompting for different information at each step (Figure 6). Initially the user is prompted for details such as project name that is used to construct a unique $\log$ in identification, an output path can also be designated so any data generated can be sent to a server location. The user is then prompted to choose analysis conditions (eg, polarity, chromatographic conditions) from a list of available methods, this is used to run the submitted analytical batch. Optimization and analytical run lists can then be imported into the console from an external server. OpenLynx ${ }^{\mathrm{TM}}$ instructs the user to place sample plates in a specific tray in the autosampler prior to finishing the log in procedure. Additional users simply start the process again.

\section{Waters instrument optimization procedure}

The OpenLynx optimization procedure is performed via loop injections of a standard solution of each compound onto the chromatography system used for sample analysis (5 min per injection). OpenLynx ${ }^{\mathrm{TM}}$ uses the molecular formula imported at $\log$ in to create a mass window for a parent ion of interest ( $\pm 25 \mathrm{amu}$ ). The software optimizes cone voltage (via a voltage ramp) on the parent mass of the compound of interest in both positive and negative ion mode. The polarity and cone voltage in which the parent ion gives the most intense response is saved by the software and a product ion scan is run across a collision energy voltage ramp. Parent ion, cone voltage, product ion and collision energy are all saved as a MassLynx ${ }^{\mathrm{TM}}$ MS method file for future analysis. The optimization process requires three injections of the standard solution totaling 15 minutes per compound. However, optimization can be done in a single injection ${ }^{11}$ by instructing the system to only operate in a single ionization polarity and by relying on the molecular formula imported at $\log$ in to yield a $\mathrm{M} \pm 1$ amu parent ion for positive and negative ion mode respectively. The software then performs a product ion scan in the appropriate polarity with a generic cone voltage and optimizes collision energy; this reduces optimization time to 5 minutes. A further time saving can be made by removing the column from the chromatographic system and making a loop injection straight into the source. It has been found that injection through the chromatographic system yields more data points throughout the 'chromato- graphic' peak, this allows the data system to characterize the peak better and gives rise to less optimization failures than loop injection straight to the mass spectrometer source. Three injections per optimization is standard. The analytical systems are exposed to a diverse range of chemical series, optimization success rate is increased by offering a choice of ionization polarity.

OpenLyn ${ }^{\mathrm{TM}}$ uses the parameters derived from instrument tuning to construct multiple reaction monitoring (MRM) methods for use in the analytical batch.

\section{Waters Corporation instrument data processing}

OpenLynx ${ }^{\mathrm{TM}}$ auto-processes all analytical batches prior to saving them on an external server. It uses a predefined calibration standard (identified by the user in the analytical batch upload) to set peak integration parameters and applies them to the entire batch. It then saves the processed data as a QuanLynx ${ }^{\mathrm{TM}}$ datafile (.qld file) in the location specified at $\log$ in.

Users can access the .qld file at office workstations via a software package called QuanLynx ${ }^{\mathrm{TM}}$ browser (Figure 7). QuanLynx $^{\mathrm{TM}}$ browser is similar in style and operation to QuickCalc ${ }^{\mathrm{TM}}$ and offers the usual versatility needed to perform quantitative bioanalytical analysis.

Quantitative results are exported to Excel prior to any secondary data processing (pharmacokinetic data analysis) and subsequent reporting of data.

\section{System suitability and quality control}

The open access systems normally run 24 hours a day 7 days a week. It is necessary to run quality control samples to monitor the mass spectrometers response and the chromatographic peak shape. Four commercially available compounds (Table 1) were selected that encompass the chemical scope that our samples fall into covering both positive and negative ionization modes. The quality control test solution is run daily and monitored to ensure consistent instrument performance (chromatographic retention time and mass spectrometer sensitivity). Instrument failures can be spotted prior to total system failure enabling preventative maintenance and planned downtime.

\section{Limitations of the open access systems}

Compounds do fail to optimize on the open access systems due a variety of reasons including, most commonly poor mass spectral sensitivity, adduct formation or in-source 

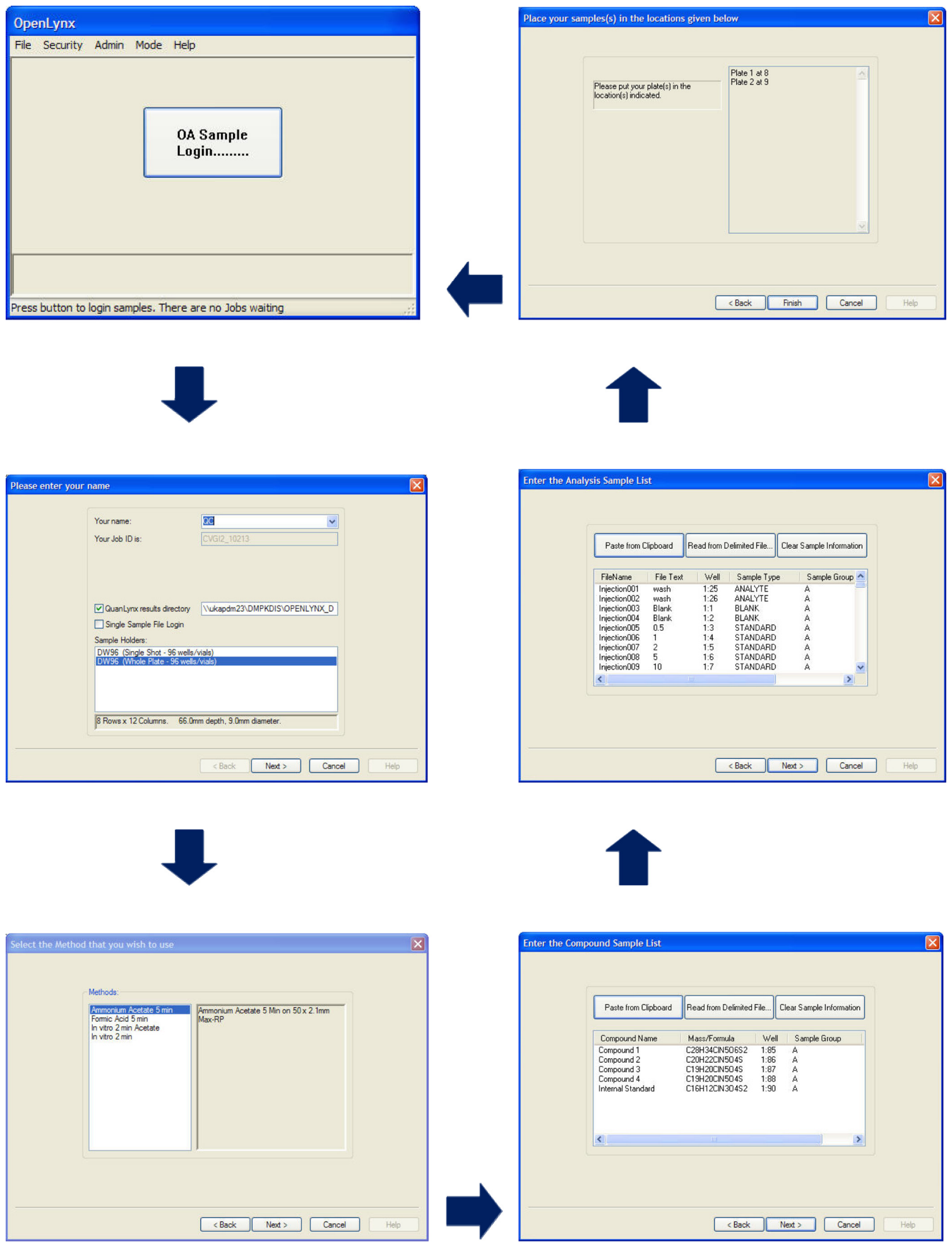

Figure 6 A schematic of the Openlynx ${ }^{T M} \log$ in process. A) The Openlynx ${ }^{T M}$ welcome screen, B) Batch identification and output screen, C) Analytical method selection screen, D) Optimization run list import screen, E) Analysis run list import screen, F) 96 well plate location instructions (generated by the software).

fragmentation. Failures can more often than not be corrected by running in an alternative solvent system, although on occasion it is necessary to consider alternative approaches, such as monitoring for an adduct or a in source fragment loss.
Chromatographic system blockages were initially a persistent problem. Preventative maintenance measures were introduced to minimize the problems, these included introducing in-line post injection filters that are changed twice 


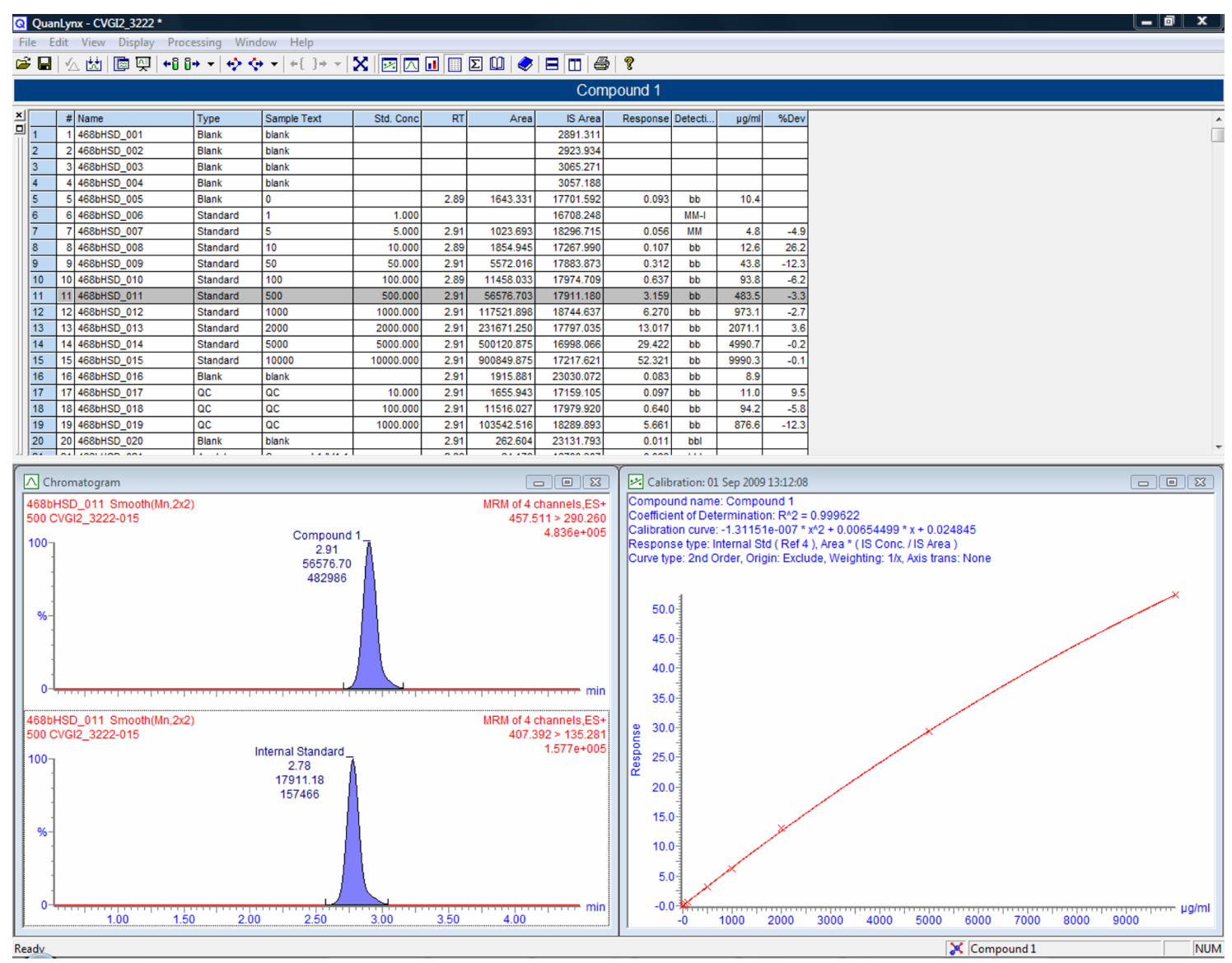

Figure 7 The QuanLynx ${ }^{\text {TM }}$ browser user interface used to auto process data from the waters corporation instruments.

weekly and guard column changes on a weekly basis. Planned maintenance cycles are carried out on the mass spectrometer hardware and peripherals twice yearly to ensure consistent performance.

Chromatographic peak co-elution could occur occasionally during analysis of cassette samples, as a consequence of the non-optimized generic gradient conditions. Problems with ion suppression caused by co-eluting peaks have been largely eliminated by the dilution of the samples with water (1:5) as part of our generic sample preparation method. Typical lower limits of quantitation are at a level of $1 \mathrm{ng} / \mathrm{mL}$ which is sufficient for pharmacokinetic profiling.

The two OA platforms discussed here both have different strengths and weaknesses. The OpenLynx ${ }^{\mathrm{TM}}$ platform instructs the operator to locate the injection plate in a specific position in the autosampler. QuickQuan ${ }^{\mathrm{TM}}$ does not specify the plate position, this must be carefully managed to ensure the same sample location is not used by different users. The QuickQuan ${ }^{\mathrm{TM}}$ platform offers a significantly faster and more comprehensive optimization procedure compared to OpenLynx ${ }^{\mathrm{TM}}$ as polarity and all tune parameters are optimized in 1 minute infusion. A full optimization on the OpenLyn ${ }^{\mathrm{TM}}$ platform requires three injections, one for each source polarity and one for product ion parameters. As mentioned earlier the number of injections can be reduced by selecting a single polarity and reducing the number of instrument parameters tuned.

Table I Quality control compounds used to monitor chromatographic and mass spectrometer performance between analytical batches

\begin{tabular}{llllll}
\hline Compound & $\begin{array}{l}\text { Polarity } \\
(+/-)\end{array}$ & $\begin{array}{l}\text { Parent } \\
\text { mass } \\
(\mathbf{M} \pm 1)\end{array}$ & $\begin{array}{l}\text { Production } \\
\text { mass }\end{array}$ & $\begin{array}{l}\text { Retention } \\
\text { time } \\
\text { (mins) }\end{array}$ & $\begin{array}{l}\text { Typical } \\
\text { area } \\
\text { (arbitary } \\
\text { units) }\end{array}$ \\
\hline Erythromycin + & 734.5 & 158.1 & 2.9 & 8613 \\
Lidocaine + & 235.2 & 86.1 & 2.8 & 24477 \\
Propanolol + & 260.2 & 116.1 & 2.7 & 169614 \\
Warfarin + & 309.1 & 163.0 & 2.5 & 121070 \\
Warfarin & - & 307.1 & 161.0 & 2.5 & 385625 \\
\hline
\end{tabular}




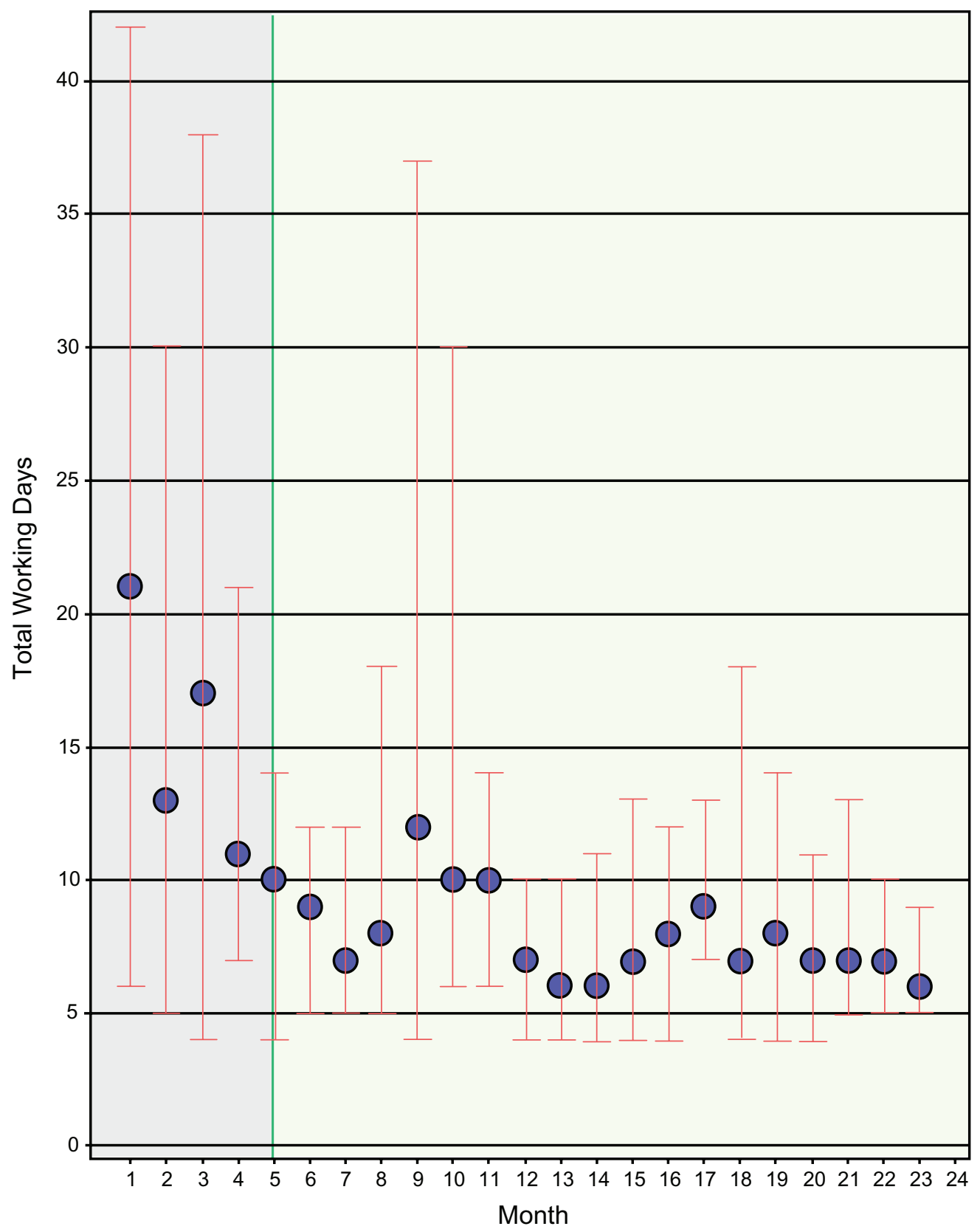

Figure 8 The average rat pharmacokinetic study turnaround times before (grey area) and after (green area) the introduction of the open access instruments (green line). The error bars denote the range of turnaround times within each dataset.

\section{Impact on pharmacokinetic study turnaround times}

The data gathered throughout the LSS project aimed at lowering rat pharmacokinetic study turnaround times demonstrated that implementation of the changes to the process, including the introduction of the open access systems, showed an immediate and marked improvement. Between 10 to 16 studies were performed each month within the 'measure' phase ( 1 to 5 months) and the 'control' phase (6 to 23 months). The average turnaround time for these studies was reduced from an initial range of between 4 to 44 working days to a desired target mean value of 10 working days. The variability was also observed to be reduced (Figure 8 ). The data collected during the 'control' phase shows that the results have been sustained for 17 months post implementation.

\section{Conclusions}

The four quantitative open access instruments have created a powerful LC-MS/MS platform. They are used for a wide variety of in vitro and in vivo DMPK assays, typically yield- 
ing specific and sensitive data in a high throughput (2 to 5 minutes per injection) environment. In addition to this they are capable of analyzing compounds with a wide range of physical properties enabling support of lead optimization projects across our research area.

We have demonstrated that open access systems have had a positive effect on the turnaround times of both in vivo and in vitro studies by utilizing mass spectrometer time more effectively and by minimizing instrument downtime. The Lean Six Sigma control phase data supports this sustained improvement.

The open access philosophy adopted by the department has been shown to provide the level of MS resource needed to perform discovery DMPK analysis in a harsh economic climate. In terms of performance metrics, during a 2 month period one open access system optimized 192 compounds and analyzed 15,294 injections, this equated to the mass spectrometer being used for 58 days out of a possible 67 (or $87 \%$ of the available time). The OA systems have allowed the DMPK scientists to spend less time on laboratory activities and more time on data interpretation and project support activities.

\section{Acknowledgments}

The authors would like to acknowledge the contribution of Graham Foster and David Temesi who are both AstraZeneca colleagues working in the cancer and infection research area, for helping set up the OpenLynx ${ }^{\mathrm{TM}}$ systems within our laboratories. We would also like to thank Mark Harrison and Jim Koers, both from Thermo Fisher Scientific, for their help turning the QuickQuan ${ }^{\mathrm{TM}}$ software into an open access platform.

\section{Disclosures}

All authors are employees of AstraZeneca.

\section{References}

1. Kola I, Landis J. Can the pharmaceutical industry reduce attrition rates. Nat Rev Drug Discov. 2004;3(8):711-716.

2. Borman S. Solid-phase synthesis, informatics, and high-throughput screening advances help combinatorial chemistry find leads. Chem Eng News. 1999;77(10):33-48.

3. Gilbert JD, Olah TV, Mcloughlin DA. In: Snyder AP, ed. Biological and Biotechnological Applications of Electrospray Ionization MS. New York, USA: The American Chemical Society; 1996:330-350.

4. Hammond C, O'Donnell CJ. Lean six sigma: its application to drug discovery. Drug Discovery World. 2008; Spring:9-18.

5. Coddington A, Van Antwerp J, Ramjit H. Critical considerations for high-reliability open access LC/MS. J Liq Chromatogr Relat Technol. 2003;26(17):2839-2859.

6. Mallis LM, Sarkahian AB, Kulishoff JM, Watts WLJ. Open-access liquid chromatography/mass spectrometry in a drug discovery environment. Mass Spectrom. 2002;37:889-896.

7. Wright P, Chassaing C, Cussans N, et al. Evolution of an open-access quantitative bioanalytical mass spectrometry service in a drug discovery environment. Biomed Chromatogr. 2006;20:585-596.

8. Ayrton J, Dear GJ, Leavens WJ, Mallett DN, Plumb RS. Use of generic fast gradient liquid chromatography-tandem mass spectroscopy in quantitative bioanalysis. J Chromatog B. 1998;709:243-254.

9. Jemal M, Xia YQ. The need for adequate chromatographic separation in the quantitative determination of drugs in biological samples by high performance liquid chromatography with tandem mass spectrometry. Rapid Commun Mass Spectrom. 1999;13:97-106.

10. Brockman A, Yan Z, Lu C, Wu JT, Elvebak L. Validation of a highthroughput absorption, distribution, metabolism and excretion (ADME) system and results for 60 literature compounds. Rapid Commun. Mass Spectrom. 2005;19:1191-1199.

11. Hiller DL, Zuzel TJ, Williams JA, Cole RO. Rapid scanning technique for the determeniation of optimal tandem mass spectrometric conditions for quantitative analysis. Rapid Commun Mass Spectrom. 1997;11:593-597.
International Journal of High Throughput Screening

\section{Publish your work in this journal}

International Journal of High Throughput Screening is an international, peer-reviewed, open access journal publishing original research, reports, editorials, reviews and commentaries dedicated to all aspects of high throughput screening, especially related to drug discovery and associated areas of biology and chemistry. The manuscript management

\section{Dovepress}

system is completely online and includes a very quick and fair peerreview system. Visit http://www.dovepress.com/testimonials.php to read real quotes from published authors. 